\title{
Die Bethe-Goldstone-Gleichung bei geschwindigkeitsabhängigen Potentialen
}

\author{
Von M. WeIgeL \\ Institut für Theoretische Physik der Universität Frankfurt am Main
}

(Z. Naturforschg. 19 a, 1268-1273 [1964]; eingegangen am 23. Juli 1964)

\begin{abstract}
The two-nucleon potential is assumed to be a quadratic function of momentum: $v=v_{1}(r)$ $+\boldsymbol{p} v_{2}(r) \boldsymbol{p}$. The Bethe-Goldstone equation $(l=0)$ has been solved for two different choices of $v$. An analytical, approximate solution is obtained.
\end{abstract}

Um das Verhalten der Streuphasen in der $\mathrm{Nu}$ kleon-Nukleon-Streuung wiederzugeben, kann man entweder ein Potential mit einem „hard core“ oder ein geschwindigkeitsabhängiges Potential ${ }^{1-5}$ verwenden. Auch die Energie pro Nukleon in Kernmaterie wurde sowohl für geschwindigkeitsabhängige Kräfte ${ }^{6}$ als auch für ein „hard core“-Potential plus anziehender Wechselwirkung ${ }^{7,8}$ berechnet.

Auf Grund des Pauli-Prinzips erwartet man für die Lösungen der Bethe-Goldstone-Gleichung nur dann größere Abweichungen gegenüber dem wechselwirkungsfreien Fall, wenn das Potential einen größeren Impulsaustausch oberhalb der FermiGrenze ermöglicht. Außerdem ist die Abweichung auf kleine Abstände beschränkt. Im Falle des „hard core“ weichen die Lösungen der Bethe-GoldstoneGleichung für kleine Abstände stark von den freien Lösungen ab; außerhalb der sogen. „healing distance" gehen sie in dieselben über.
Es soll das Verhalten der Lösungen der BetheGoldstone-Gleichung untersucht werden, wenn man geschwindigkeitsabhängige Potentiale verwendet.

Man hat zwei äquivalente Möglichkeiten für ein rotationsinvariantes, höchstens quadratisch vom Impuls abhängiges Potential:

$$
\left\langle\boldsymbol{x}|v| \boldsymbol{x}^{\prime}\right\rangle=\left\langle\boldsymbol{x}\left|\left(v_{1}(|\boldsymbol{x}|)+\frac{\lambda}{m} \boldsymbol{p} v_{2}(|\boldsymbol{x}|) \boldsymbol{p}\right)\right| \boldsymbol{x}^{\prime}\right\rangle
$$

oder

$$
\begin{array}{r}
\left\langle\boldsymbol{x}|\tilde{v}| \boldsymbol{x}^{\prime}\right\rangle=\langle\boldsymbol{x}|\left(\tilde{v}_{1}(|\boldsymbol{x}|)+\frac{\lambda}{m}\left(^{2} \tilde{v}_{\mathbf{2}}(|\boldsymbol{x}|)\right.\right. \\
\left.\left.+\tilde{v}_{\mathbf{2}}(|\boldsymbol{x}|) \boldsymbol{p}^{2}\right)\right)\left|\boldsymbol{x}^{\prime}\right\rangle .
\end{array}
$$

Beide fallen zusammen, wenn man setzt $(r=|\boldsymbol{x}|)$ :

$v_{2}(r)=2 \tilde{v}_{2}(r)$,

$v_{1}(r)=\tilde{v}_{1}(r)-\frac{\lambda}{m}\left(\frac{2}{r} \frac{\mathrm{d}}{\mathrm{d} r} \tilde{v}_{2}(r)+\frac{\mathrm{d}^{2}}{\mathrm{~d} r^{2}} \tilde{v}_{2}(r)\right)$.

\section{Bethe-Goldstone-Gleichung}

Nach Bethe und Goldstone gilt für die Reaktionsmatrix ${ }^{7}$ :

$$
\left\langle\boldsymbol{k}|G| \boldsymbol{k}_{0}, \boldsymbol{P}\right\rangle=\left\langle\boldsymbol{k}|v| \boldsymbol{k}_{0}\right\rangle+(2 \pi)^{-3} P \int \mathrm{d} \boldsymbol{k}^{\prime} \frac{\left\langle\boldsymbol{k}|v| \boldsymbol{k}^{\prime}\right\rangle\left\langle\boldsymbol{k}^{\prime}|G| \boldsymbol{k}_{0}, \boldsymbol{P}\right\rangle}{\varepsilon\left(\boldsymbol{P}, \boldsymbol{k}^{\prime}, \boldsymbol{k}_{0}\right)} .
$$

Dabei ist $2 \boldsymbol{P}$ der Schwerpunktsimpuls. Die Integration über $\boldsymbol{k}^{\prime}$ ist so zu verstehen, daß $\left|\boldsymbol{P} \pm \boldsymbol{k}^{\prime}\right|$ größer als $k_{\mathrm{F}}$ ist. Außerdem gilt $\left|\boldsymbol{P} \pm \boldsymbol{k}_{0}\right|<k_{\mathrm{F}} \quad\left(k_{\mathrm{F}}\right.$ Fermi-Impuls $)$.

$$
\varepsilon\left(\boldsymbol{P}, \boldsymbol{k}, \boldsymbol{k}_{0}\right)=E\left(\boldsymbol{P}+\boldsymbol{k}_{0}\right)+E\left(\boldsymbol{P}-\boldsymbol{k}_{0}\right)-E(\boldsymbol{P}+\boldsymbol{k})-E(\boldsymbol{P}-\boldsymbol{k}),
$$

$E(\boldsymbol{k})$ ist die Energie des Nukleons mit dem Impuls $\boldsymbol{k}$.

Führen wir jetzt die Wellenfunktion $\chi$ durch die Beziehung $G=v \chi$ ein, so gilt für dieselbe im Ortsraum:

$$
\chi\left(\boldsymbol{x}, \boldsymbol{k}_{0}, \boldsymbol{P}\right)=\exp \left(i \boldsymbol{k}_{0} \boldsymbol{x}\right)+\int \mathrm{d} \boldsymbol{x}^{\prime} \mathrm{d} \boldsymbol{x}^{\prime \prime} \Gamma\left(\boldsymbol{x}-\boldsymbol{x}^{\prime}\right)\left\langle\boldsymbol{x}^{\prime}|v| \boldsymbol{x}^{\prime \prime}\right\rangle \chi\left(\boldsymbol{x}^{\prime \prime}, \boldsymbol{k}_{0}, \boldsymbol{P}\right),
$$

1 J. S. Levinger, Proc. Int. Conf. Nuclear Structure (Kingston 1960), North-Holland Publ. Co., Amsterdam 1960.

2 M. Razavy, G. Field u. J. S. Levinger, Phys. Rev. 125, 269 [1962].

3 O. Rojo u. L. M. Simmons, Phys. Rev. 125, 273 [1962].

${ }^{4}$ A. M. Green, Nucl. Phys. 33, 218 [1962].
5 E. W ERner, Nucl. Phys. 35, 324 [1962].

6 F. Peischl u. E. Werner, Nucl. Phys. 43, 372 [1963].

7 H. A. Bethe u. J. Goldstone, Proc. Roy. Soc., Lond. A 238, New York 3, 241 [1958]. 551 [1957].

8 L. C. Gomes, J. D. Walecka u. V. F. Weisskopf, Ann. Phys.. 
wobei

$$
\Gamma\left(\boldsymbol{x}-\boldsymbol{x}^{\prime}\right)=\frac{1}{(2 \pi)^{3}} P \int \mathrm{d} \boldsymbol{k} \exp \left\{i \boldsymbol{k}\left(\boldsymbol{x}-\boldsymbol{x}^{\prime}\right)\right\} \frac{\Theta\left(|\boldsymbol{P}+\boldsymbol{k}|-k_{\mathrm{F}}\right) \Theta\left(|\boldsymbol{P}-\boldsymbol{k}|-k_{\mathrm{F}}\right)}{\varepsilon\left(\boldsymbol{P}, \boldsymbol{k}, \boldsymbol{k}_{0}\right)} ;
$$

$\Theta(x)$ ist die bekannte Sprungfunktion $(\Theta(x)=1$ für $x>0 ; \Theta(x)=0$ für $x<0)$.

Da es uns auf das prinzipielle Verhalten der Wellenfunktion ankommt, können wir $\boldsymbol{P}=\boldsymbol{O}$ setzen. Dann separiert Gl. (5) in Polarkoordinaten, und für den radialen Anteil erhält man mit dem Potential (1) folgende Gleichung:

$$
R_{l}\left(r, k_{0}\right)=j_{l}\left(k_{0} r\right)+\int_{0}^{\infty} A_{l}\left(r, r^{\prime} k_{0}\right) V_{l}\left(r^{\prime}\right) R_{l}\left(r^{\prime}, k_{0}\right) \mathrm{d} r^{\prime} .
$$

Dabei ist $(\hbar=1)$ :

$$
j_{l}(x)=x^{l}\left(-\frac{1}{x} \frac{\mathrm{d}}{\mathrm{d} x}\right)^{l} \frac{\sin x}{x},
$$

$$
\begin{gathered}
V_{l}(r)=v_{1}(r)-\frac{\lambda}{m}\left(\left(\frac{\mathrm{d}}{\mathrm{d} r} v_{2}(r)\right) \frac{\mathrm{d}}{\mathrm{d} r}+v_{2}(r)\left(\frac{\mathrm{d}^{2}}{\mathrm{~d} r^{2}}+\frac{2}{r} \frac{\mathrm{d}}{\mathrm{d} r}-\frac{l(l+1)}{r^{2}}\right)\right), \\
A_{l}\left(r, r^{\prime}, k_{0}\right)=\frac{2}{\pi} P \int_{k_{\mathrm{F}}}^{\infty} r^{\prime 2} k^{2} \mathrm{~d} k \frac{j_{l}(k r) j_{l}\left(k r^{\prime}\right)}{\varepsilon\left(\boldsymbol{O}, \boldsymbol{k} \boldsymbol{k}_{0}\right)} .
\end{gathered}
$$

Führt man jetzt die effektive Massenapproximation ein, so kann man leicht Gl. (7) in eine Integrodifferentialgleichung verwandeln.

Mit

$$
\begin{gathered}
\varepsilon\left(\boldsymbol{O}, \boldsymbol{k}, \boldsymbol{k}_{0}\right)=\frac{1}{m^{*}}\left(k_{0}^{2}-k^{2}\right) \quad \text { erhält man: } \\
\left\{\frac{\mathrm{d}^{2}}{\mathrm{~d} r^{2}}+\frac{2}{r} \frac{\mathrm{d}}{\mathrm{d} r}+k_{0}^{2}-\frac{l(l+1)}{r^{2}}-m^{*} V_{l}(r)\right\} R_{l}\left(r, k_{0}\right)=-m^{*} \int_{0}^{\infty} K_{l}\left(r, r^{\prime}\right) V_{l}\left(r^{\prime}\right) R_{l}\left(r^{\prime}, k_{0}\right) \mathrm{d} r^{\prime},
\end{gathered}
$$

wobei

$$
K_{l}\left(r, r^{\prime}\right)=\frac{2}{\pi} \int_{0}^{k_{F}} r^{\prime 2} k^{2} j_{l}(k r) j_{l}\left(k r^{\prime}\right) \mathrm{d} k .
$$

\section{Behandlung spezieller Potentiale}

Ausgehend von Gl. (7) und (9) wollen wir uns auf solche Potentiale beschränken, die bei der Streuung im Vakuum eine explizite Lösung zulassen. Man kann vermuten, daß für kleine Abstände diese Lösungen eine gute erste Approximation darstellen und auf dieser Basis (7) bzw. (9) näherungsweise lösen. Weiterhin ist bekannt ${ }^{8}$, daß nichtsinguläre, lokale Wechselwirkungen nur geringfügige Abweichungen bedingen, und wir wollen deshalb außerhalb der „Reichweite“ von $v_{2}(r)$ das lokale Potential vernachlässigen.

Als Potential wählen wir ${ }^{2}$ :

$$
v_{1}(r)=-V_{0} \Theta(b-r)-g \frac{\lambda}{m} \frac{\delta(r-b)}{r}, \quad v_{2}(r)=-\Theta(b-r) .
$$

Das Potential legt folgende Aufspaltung nahe ( $k_{0}$ unterdrückt) :

$$
R_{l}(r)=\Theta(r-b) F_{l}(r)+\Theta(b-r) G_{l}(r) .
$$

Die Beziehung (9) lautet damit $\left[G_{l}(b)=F_{l}(b)\right]$ :

$$
\begin{gathered}
\delta(r-b)\left(\frac{\mathrm{d}}{\mathrm{d} r}\left(F_{l}(r)-G_{l}(r)+\lambda^{*} G_{l}(r)\right)+g \frac{\lambda^{*}}{2 r}\left(G_{l}(r)+F_{l}(r)\right)\right)+\Theta(r-b) L\left(r, k_{0}\right) F_{l}(r) \\
+\left(1-\lambda^{*}\right) \Theta(b-r) L\left(r, k^{\prime}\right) G_{l}(r) \\
=-\int_{0}^{l} \mathrm{~d} r^{\prime} K_{l}\left(r, r^{\prime}\right)\left(\left(1-\lambda^{*}\right)\left(k_{0}^{2}-k^{\prime 2}\right)+\lambda^{*} L\left(r^{\prime}, k_{0}\right)\right) G_{l}\left(r^{\prime}\right)+\left.\lambda^{*} K_{l}(r, b)\left(\frac{\mathrm{d}}{\mathrm{d} r^{\prime}}+\frac{g}{b}\right) G_{l}\left(r^{\prime}\right)\right|_{r^{\prime}=b},
\end{gathered}
$$

wobei

$$
\lambda^{*}=\frac{m^{*}}{m} \lambda ; \quad k^{\prime 2}=\frac{m^{*} V_{0}+k_{0}{ }^{2}}{1-\lambda^{*}} ; \quad L(r, k) \equiv \frac{\mathrm{d}^{2}}{\mathrm{~d} r^{2}}+\frac{2}{r} \frac{\mathrm{d}}{\mathrm{d} r}+k^{2}-\frac{l(l+1)}{r^{2}} .
$$


Beziehung (13) ergibt die Randbedingungen bei $r=b$ :

$$
F_{l}(b)=G_{l}(b) ;\left.\quad \frac{\mathrm{d}}{\mathrm{d} r} F_{l}(r)\right|_{r=b}=\left.\left(1-\lambda^{*}\right) \frac{\mathrm{d}}{\mathrm{d} r} G_{l}(r)\right|_{r=b}-\frac{g}{b} \lambda^{*} G_{l}(b) .
$$

Für die Streuung im Vakuum $\left(k_{\mathrm{F}}=0\right)$ kann man (13) exakt lösen ${ }^{2}$. Es ergibt sich:

$$
R_{l \mathrm{v}}(r)=\Theta(b-r) d_{l} j_{l}\left(k^{\prime} r\right)+\Theta(r-b)\left(j_{l}\left(k_{0} r\right)-\operatorname{tg} \delta_{l} n_{l}\left(k_{0} r\right)\right) .
$$

Die Konstanten $d_{l}$ und tg $\delta_{l}$ ergeben sich aus den Randbedingungen (15).

Wir beschränken uns auf den Fall $l=0$. Hierfür ist es zweckmäßiger, die Funktion $u(r)=r R_{0}(r)$ zu betrachten:

$$
u(r)=\Theta(b-r) u_{2}(r)+\Theta(r-b) u_{1}(r) .
$$

Diese Funktion genügt folgender Gleichung:

$$
\begin{aligned}
& \Theta(b-r) u_{2}(r)+\Theta(r-b) u_{1}(r)= \\
& \frac{\sin k_{0} r}{k_{0}}+\int_{0}^{b} \mathrm{~d} r^{\prime} \frac{r}{r^{\prime}} \frac{A_{0}\left(r, r^{\prime}\right)}{m^{*}}\left(-m^{*} V_{0}+\lambda^{*} \frac{\mathrm{d}^{2}}{\mathrm{~d} r^{\prime 2}}\right) u_{2}\left(r^{\prime}\right)-\left.\frac{\lambda^{*}}{m^{*}} \frac{r}{b} A_{0}(r, b)\left(\frac{\mathrm{d}}{\mathrm{d} r^{\prime}}-\frac{1}{r^{\prime}}(1-g)\right) u_{2}\left(r^{\prime}\right)\right|_{r^{\prime}=b} .
\end{aligned}
$$

Die Randbedingungen bei $r=b$ lauten:

$$
u_{1}(b)=u_{2}(b) ;\left.\quad \frac{\mathrm{d}}{\mathrm{d} r} u_{1}(r)\right|_{r=b}=\left.\left(1-\lambda^{*}\right) \frac{\mathrm{d}}{\mathrm{d} r} u_{2}(r)\right|_{r=b}+\frac{\lambda^{*}}{b}(1-g) u_{2}(b) .
$$

Gl. (18) bedingt das bekannte asymptotische Verhalten $\left(k_{\mathrm{F}} \neq 0\right)$ :

$$
\lim _{r \rightarrow \infty}\left(u_{1}(r)-\frac{\sin k_{0} r}{k_{0}}\right)=0 .
$$

Durch Anwendung des Operators $\left(\mathrm{d}^{2} / \mathrm{d} r^{2}\right)+k_{0}{ }^{2}$ und partielle Integration des Integrals der rechten Seite ergibt sich aus (18) folgende Integrodifferentialgleichung für $u(r)$ :

$$
\begin{aligned}
& \Theta(r-b)\left(\frac{\mathrm{d}^{2}}{\mathrm{~d} r^{2}}+k_{0}{ }^{2}\right) u_{1}(r)+\left(1-\lambda^{*}\right) \Theta(b-r)\left(\frac{\mathrm{d}^{2}}{\mathrm{~d} r^{2}}+k^{\prime 2}\right) u_{2}(r) \\
& =\left(-m^{*} V_{0}+\lambda^{*} \frac{\mathrm{d}^{2}}{\mathrm{~d} r^{2}}\right) \int_{0}^{b} M\left(r, r^{\prime}\right) u_{2}\left(r^{\prime}\right) \mathrm{d} r^{\prime}-\left.\lambda^{*} u_{2}(b)\left(\frac{\mathrm{d}}{\mathrm{d} r^{\prime}}-\frac{1}{b}(1-g)\right) M\left(r, r^{\prime}\right)\right|_{r^{\prime}=b}, \\
& \text { wobei } \\
& \qquad\left(r, r^{\prime}\right)=-\frac{1}{\pi} \int_{-k_{\mathrm{F}}}^{+k_{F}} \sin k r \sin k r^{\prime} \mathrm{d} k
\end{aligned}
$$

ist. Benutzt man

$$
\frac{2}{\pi} P \int_{0}^{\infty} \frac{\sin k r \sin k r^{\prime}}{k^{2}-k_{0}{ }^{2}} \mathrm{~d} k=\frac{1}{k_{0}}\left(\Theta\left(r-r^{\prime}\right) \cos k_{0} r \sin k_{0} r^{\prime}+\Theta\left(r^{\prime}-r\right) \sin k_{0} r \cos k_{0} r^{\prime}\right),
$$

so kann man (18) auf folgende Weise umschreiben:

$$
\begin{aligned}
& u_{1}(r)=\frac{\sin k_{0} r}{k_{0}}-\frac{\left(1-\lambda^{*}\right)\left(k_{0}{ }^{2}-k^{\prime 2}\right)}{\mathrm{k}_{0}} \cos k_{0} r \int_{0}^{b} \sin k_{0} r^{\prime} u_{2}\left(r^{\prime}\right) \mathrm{d} r^{\prime} \\
& \quad+\lambda^{*} u_{2}(b)\left(\cos k_{0} r \cos k_{0} b-\frac{(1-g)}{k_{0} b} \cos k_{0} r \sin k_{0} b\right)+\int_{\sigma}^{b} P_{1}\left(r, r^{\prime}\right) u_{2}\left(r^{\prime}\right) \mathrm{d} r^{\prime}+\lambda^{*} u_{2}(b) P_{2}(r, b), \\
& \left(1-\lambda^{*}\right) u_{2}(r)=\frac{\sin k_{0} r}{k_{0}}-\left(1-\lambda^{*}\right)\left(k_{0}{ }^{2}-k^{\prime 2}\right)\left(\cos k_{0} r \int_{0}^{r} \frac{\sin k_{0} r^{\prime}}{k_{0}} u_{2}\left(r^{\prime}\right) \mathrm{d} r^{\prime}+\frac{\sin k_{0} r}{k_{0}} \int_{r}^{b} \cos k_{0} r^{\prime} u_{2}\left(r^{\prime}\right) \mathrm{d} r^{\prime}\right) \\
& -\lambda^{*} u_{2}(b)\left(\sin k_{0} r \sin k_{0} b+\frac{(1-g)}{k_{0} b} \cos k_{0} b \sin k_{0} r\right)+\int_{0}^{b} \mathrm{~d} r^{\prime} P_{1}\left(r, r^{\prime}\right) u_{2}\left(r^{\prime}\right)+\lambda^{*} u_{2}(b) P_{2}(r, b) .
\end{aligned}
$$


Dabei ist:

$$
\begin{aligned}
& P_{1}\left(r, r^{\prime}\right)=\frac{2}{\pi} P \int_{0}^{k \mathrm{~F}}\left(\lambda^{*}\left(k^{2}-k_{0}^{2}\right)+\left(1-\lambda^{*}\right)\left(k^{\prime 2}-k_{0}{ }^{2}\right)\right) \frac{\sin k r \sin k r^{\prime}}{k_{0}{ }^{2}-k^{2}} \mathrm{~d} k, \\
& P_{2}\left(r, r^{\prime}\right)=\frac{2}{\pi} P \int_{0}^{k_{\mathrm{F}}}\left(k \cos k r^{\prime}-\frac{(1-g)}{b} \sin k r^{\prime}\right) \frac{\sin k r}{k_{0}{ }^{2}-k^{2}} \mathrm{~d} k .
\end{aligned}
$$

Aus den Gln. (24) und (25) geht hervor, daß bis auf Quadratur nur die Integralgleichung (24) im Bereich $[0, b]$ gelöst werden muß. Es ist naheliegend ${ }^{8}$, daß für kleine Abstände die Lösung des Vakuumstreuproblems eine gute Approximation darstellt. Dieselbe erfüllt auch die Randbedingung (19) und ist nach (16) explizit bekannt:

$$
\begin{aligned}
u_{\mathrm{v}}(r) & =\Theta(b-r) d_{0} \frac{\sin k^{\prime} r}{k^{\prime}} \\
& +\Theta(r-b)\left(\frac{\sin k_{0} r}{k_{0}}+\frac{\operatorname{tg} \delta_{0}}{k_{0}} \cos k_{0} r\right) .
\end{aligned}
$$

Die Konstanten $d_{0}$ und $\operatorname{tg} \delta_{0}$ bestimmen sich aus (19).

Als erste Approximation führe ich in (24) und (25) auf der rechten Seite $u_{2 \mathrm{v}}(r)$ ein. Die Integrationen lassen sich elementar durchführen.

Es ergibt sich:

$$
\begin{aligned}
& u_{1}{ }^{(1)}(r)=\frac{\sin k_{0} r}{k_{0}}+\frac{\operatorname{tg} \delta_{0}}{k_{0}} \cos k_{0} r+F(r), \\
& u_{2}{ }^{(1)}(r)=d_{0} \frac{\sin k^{\prime} r}{k^{\prime}}+\frac{1}{1-\lambda^{*}} F(r) .
\end{aligned}
$$

$F(r)$ ist im Anhang angegeben.

\section{Numerische Ergebnisse}

Wir verwenden im wesentlichen die von Razavy, Field und Levinger ${ }^{2}$ vorgeschlagenen Potentialparameter. Das zweite Potential $(g=1)$ simuliert recht gut für höhere Energien die Streuphasen des "hard-core“-Potentials $\left(r_{\mathrm{c}}=0,295 f\right.$, s. Tab. 1, 2).

Gl. (24) kann man durch Iteration, beginnend mit $u_{2 \mathrm{v}}(r)$, lösen. Ein Maß für die Güte der Lösung ist die Befriedigung der Randbedingungen (19) bei $r=b$. Numerisch wurde gezeigt, daß (28) in den meisten Fällen ausreichend ist. Die Randbedingungen sind bis auf wenige Prozent erfüllt.

In Abb. 1, 2, 3 und in Tab. 3 sind die Lösungen für zwei Potentiale dargestellt. Die Abb. 1 stellt die Funktionen

$$
g(r)=u(r)-u_{0}(r) \quad \text { und } \quad g_{\mathrm{v}}(r)=u(r)-u_{\mathrm{v}}(r)
$$

für das Potential $W_{1}$ dar. Es ergibt sich praktisch der Fall eines rein anziehenden Potentials ${ }^{7}$, da das

\begin{tabular}{|l|c|c|c|c|}
\hline & $b(f)$ & $V_{0}(\mathrm{MeV})$ & $\lambda$ & $g$ \\
\hline Potential $W_{1}$ & 2,4 & 16,9 & $-0,21$ & 0,0 \\
Potential $W_{2}$ & 0,5 & 51 & $-1,64$ & 1,0 \\
\hline
\end{tabular}

Tab. 1. Potentialparameter ${ }^{2}$.

\begin{tabular}{|c|c|c|c|}
\hline $\begin{array}{c}E \\
(\mathrm{MeV})\end{array}$ & $\begin{array}{c}\delta_{0} \\
\text { Potential } W_{1}\end{array}$ & $\begin{array}{c}\delta_{0} \\
\text { Potential } W_{2}\end{array}$ & $\begin{array}{c}\delta_{0} \\
\text {,hard core“ } \\
0,295 f\end{array}$ \\
\hline 30 & 0,750 & $-0,181$ & $-0,178$ \\
60 & 0,474 & $-0,255$ & $-0,251$ \\
90 & 0,273 & $-0,311$ & $-0,308$ \\
150 & 0,002 & $-0,397$ & $-0,397$ \\
210 & $-0,141$ & $-0,465$ & $-0,470$ \\
300 & $-0,196$ & $-0,547$ & $-0,562$ \\
\hline
\end{tabular}

Tab. 2. Streuphasen $\left(l=0 ; m^{*} / m=1\right)$.

Potential nur schwach impulsabhängig ist $(|\lambda|$ ist klein). Die Abweichungen von der freien Lösung sind sehr gering, so da $\beta$ hier an sich eine Iteration beginnend mit $u_{0}(r)$ an Stelle von $u_{\mathrm{v}}(r)$ angebrachter gewesen wäre. Für das Ergebnis spielt das in diesem Falle keine Rolle. In Abb. 2 und 3 und in Tab. 3 ist für das Potential $W_{2}$ für zwei Fälle $k_{\mathrm{F}} u(r), k_{\mathrm{F}} u_{0}(r)$ und $k_{\mathrm{F}} u_{\mathrm{V}}(r)$ dargestellt. Die Lösungen zeigen das typische Verhalten eines abstoßenden Potentials ${ }^{8}$. Die Abweichungen von $u(r)$ ge-

\begin{tabular}{|c|c|c|c|c|c|c|}
\hline \multirow[b]{2}{*}{$k_{\mathrm{F}} r$} & \multicolumn{3}{|c|}{$k=0,0 k_{\mathrm{F}}$} & \multicolumn{3}{|c|}{$k=0,5 k_{\mathrm{F}}$} \\
\hline & 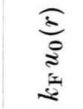 & 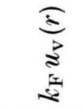 & $\underset{\substack{E \\
\Sigma}}{\Xi}$ & 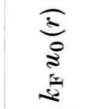 & 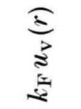 & 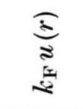 \\
\hline $0,64=k_{\mathrm{r}} b$ & 0,64 & 0,36 & 0,4 & 0.6 & 0,37 & 0,41 \\
\hline 0,80 & 0,80 & 0,52 & 0,6 & 0,78 & 0,52 & 0,60 \\
\hline 1,28 & 1,28 & 1,00 & 1,1 & 2,20 & 0,97 & 1,09 \\
\hline 2,08 & 2,08 & 1,81 & 2,09 & 1,73 & 1,59 & 1,73 \\
\hline 3,21 & 3,21 & 2,92 & 3,26 & 2,00 & 2,01 & 2,06 \\
\hline 4,65 & 4,65 & 4,37 & 4,66 & 1,46 & 1,65 & 1,47 \\
\hline 6,42 & 6,42 & 6,14 & 6,39 & $-0,13$ & 0,14 & $-0,17$ \\
\hline 8,50 & 8,50 & 8,22 & 8,51 & $-1,79$ & $-1,67$ & $-1,78$ \\
\hline 10,91 & 10,91 & 10,63 & 10,91 & $-1,47$ & $-1,66$ & $-1,47$ \\
\hline
\end{tabular}
genüber $u_{0}(r)$ betragen bei $r=b$ rund $20 \%$. In Abb. 2 ist das Verhalten der Bethe-Goldstone-Lösung $k_{\mathrm{F}} \boldsymbol{u}_{\mathrm{c}}(r)$ für das "hard core"-Potential $\left(r_{\mathrm{c}}=\right.$ $0,259 f$ ) qualitativ zum Vergleich mit angegeben. Für den Fermi-Impuls wurde der Wert $k_{\mathrm{F}}=1,28333$ $f^{-1}$ verwendet.

Tab. 3. Wellenfunktionen $\left(m^{*} / m=0,5 ; r \geqq b\right)$. 


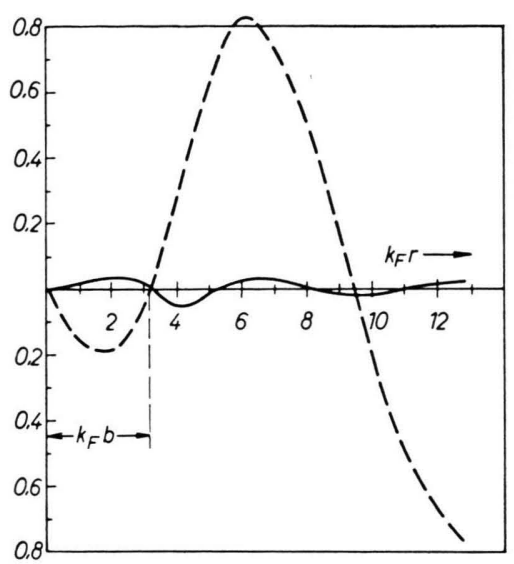

Abb. 1. $k_{\mathrm{F}} g(r)=k_{\mathrm{F}}\left[u(r)-u_{0}(r)\right]$ (durchgezogene Kurve) und $k_{\mathrm{F}} g_{\mathrm{V}}(r)$ $=k_{\mathrm{F}}\left[u(r)-u_{\mathrm{V}}(r)\right] \quad$ (gestrichelte Kurve) als Funktion von $k_{\mathrm{F}} r$ bei Verwendung des Potentials $W_{1}$. Parameter: $k_{0}=0,5 k_{\mathrm{F}} ; k=0,582$ $k_{\mathrm{F}} ; d_{0}=1,206 ; \operatorname{tg} \delta_{0}=0,391 ;$

$$
m^{*} / m=0,5 \text {. }
$$

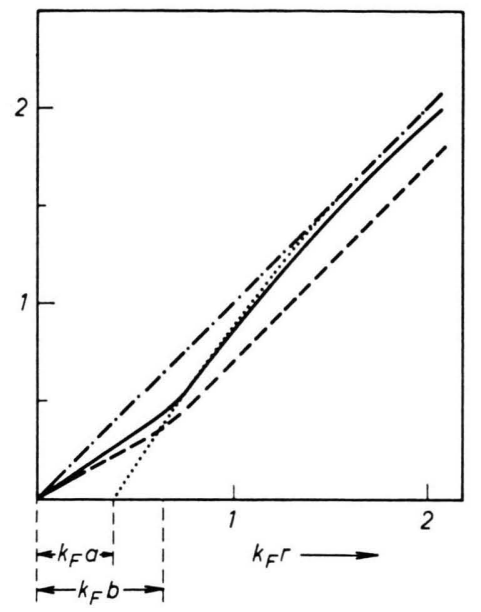

Abb. 2. Die Funktionen $k_{\mathrm{F}} u(r)$ (durchgezogene Kurve), $k_{\mathrm{F}} u_{\mathrm{V}}(r)$ (isoliertes Nukleonenpaar, gestrichelte Kurve) und $k_{\mathrm{F}} u_{0}(r)$ (keine Wechselwirkung, strichpunktierte Kurve) als Funktion von $k_{\mathrm{F}} r$ bei Verwendung des Potentials $W_{2}$. Parameter: $k_{0}=0,0 k_{\mathrm{F}} ; k^{\prime}=0,453 k_{\mathrm{F}}$; $d_{0}=0,574 ; k \mathrm{~F} / k_{0} \cdot \operatorname{tg} \delta_{0}=-0,279 ; m^{*} / m=0,5$. Außerdem ist das qualitative Verhalten der „hard core“-Lösung $k_{\mathrm{F}} u_{\mathrm{c}}(r)$ (punktierte Kurve) angegeben $\left(r_{\mathrm{c}}=0,295 f\right)$.

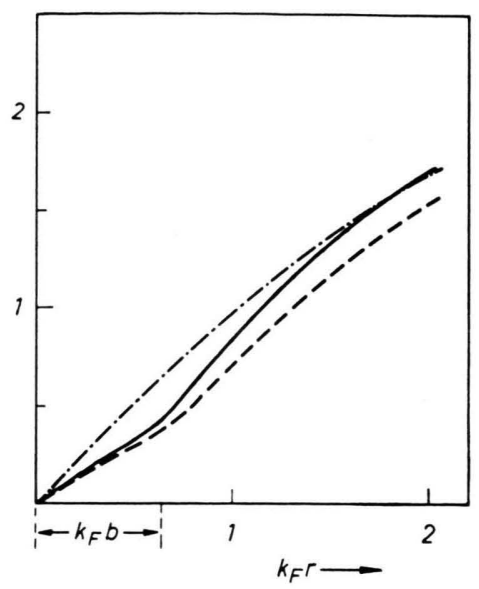

Abb. 3. Die Funktionen $k_{\mathrm{F}} u(r)$ (durchgezogene Kurve), $k_{\mathrm{F}} u_{\mathrm{v}}(r)$ (gestrichelte Kurve) und $k_{\mathrm{F}} u_{0}(r)$ (strichpunktierte Kurve) als Funktion von $k \mathrm{~F} r$ bei Verwendung des Potentials $W_{2}$. Parameter: $k_{0}=0,5$ $k_{\mathrm{F}} ; k=0,585 k_{\mathrm{F}} ; \quad d_{0}=0,586$; $\operatorname{tg} \delta_{0}=-0,139 ; m^{*} / m=0,5$.

Für fördernde Diskussionen möchte ich Herrn Prof. Dem „Deutschen Rechenzentrum“ in Darmstadt danke Dr. F. BEсK und Herrn Prof. Dr. G. Süssmann danken. ich für die Hilfe bei der numerischen Auswertung.

\section{Anhang}

$F(r)$ ist nach $(24),(25)$ und $(28)$ :

$$
\begin{aligned}
F(r) & =\frac{2}{\pi} P \int_{0}^{b} \mathrm{~d} r^{\prime} \int_{0}^{k_{\mathrm{F}}} \mathrm{d} k d_{0} \frac{\sin k^{\prime} r^{\prime}}{k^{\prime}} \frac{\lambda^{*}\left(k^{2}-k_{0}{ }^{2}\right)+\left(1-\lambda^{*}\right)\left(k^{\prime 2}-k_{0}{ }^{2}\right)}{k_{0}{ }^{2}-k^{2}} \sin k r \sin k r^{\prime} \\
& +\lambda^{*} d_{0} \frac{\sin k^{\prime} b}{k^{\prime}} \frac{2}{\pi} P \int_{0}^{k_{\mathrm{F}}} \mathrm{d} k \frac{\sin k r}{k_{0}{ }^{2}-k^{2}}\left(k \cos k b-\frac{(1-g)}{b} \sin k b\right) .
\end{aligned}
$$

Ausführung der Integrationen ergibt ${ }^{9}$ :

$$
\begin{aligned}
F(r) & =\frac{d_{0}}{\pi k^{\prime}}\left\{-\lambda^{*} \gamma\left(r, b, k^{\prime},-k^{\prime}\right)+\frac{1-\lambda^{*}}{2 k_{0}}\left(k^{\prime 2}-k_{0}{ }^{2}\right)\left[\frac { 1 } { k ^ { \prime } - k _ { 0 } } \left(\gamma\left(r, b, k_{0},-k^{\prime}\right)\right.\right.\right. \\
& \left.\left.-\gamma\left(r, b, k^{\prime},-k^{\prime}\right)\right)+\frac{1}{k^{\prime}+k_{0}}\left(\gamma\left(r, b, k^{\prime},-k^{\prime}\right)-\gamma\left(r, b,-k_{0},-k^{\prime}\right)\right)\right] \\
& \left.-\frac{\lambda^{*}}{2} \sin k^{\prime} b\left(\alpha(r+b)+\alpha(r-b)+\lambda^{*} \sin k^{\prime} b \frac{1-g}{k_{0} b} \gamma\left(r, b, k_{0}, 0\right)\right)\right\} .
\end{aligned}
$$

Dabei sind folgende Definitionen vereinbart:

$$
\begin{gathered}
\gamma\left(r_{1}, r_{2}, k_{1}, k_{2}\right)=\frac{1}{2} f_{1}\left(r_{1}, r_{2}, k_{1}\right) \cos \left(r_{2}\left(k_{1}+k_{2}\right)-r_{1} k_{1}\right)-f_{2}\left(r_{1}, r_{2}, k_{1}\right) \sin \left(r_{2}\left(k_{1}+k_{2}\right)-r_{1} k_{1}\right) \\
-f_{1}\left(-r_{1}, r_{2}, k_{1}\right) \cos \left(r_{2}\left(k_{1}+k_{2}\right)+r_{1} k_{1}\right)+f_{2}\left(-r_{1}, r_{2}, k_{1}\right) \sin \left(r_{2}\left(k_{1}+k_{2}\right)+r_{1} k_{1}\right), \\
\alpha(r)=f_{1}\left(0, r, k_{0}\right) \sin k_{0} r+f_{2}\left(0, r, k_{0}\right) \cos k_{0} r,
\end{gathered}
$$

${ }^{9}$ F. TöLKE, Praktische Funktionenlehre, Bd. I, Springer-Verlag, Berlin 1943. 


$$
\begin{aligned}
& f_{1}\left(r_{1}, r_{2}, k\right)=\operatorname{Ci}\left(\left|\left(r_{1}-r_{2}\right)\left(k_{\mathrm{F}}-k\right)\right|\right)-\operatorname{Ci}\left(\left|\left(r_{1}-r_{2}\right)\left(k_{\mathrm{F}}-k\right)\right|\right), \\
& f_{2}\left(r_{1}, r_{2}, k\right)=\operatorname{Si}\left(\left(r_{1}-r_{2}\right)\left(k_{\mathrm{F}}-k\right)\right)-\operatorname{Si}\left(\left(r_{1}-r_{2}\right)\left(k+k_{\mathrm{F}}\right)\right) .
\end{aligned}
$$

Man kann nach einigen Umformungen das richtige asymptotische Verhalten nachweisen:

$$
\lim _{r \rightarrow \infty}\left(F(r)+\frac{\operatorname{tg} \delta_{0}}{k_{0}} \cos k_{0} r\right)=0 .
$$

\title{
Zum normalen Skin-Effekt unter Berüdksidhtigung der magnetischen Widerstandsänderung
}

\author{
Von Rudolf KleiN * \\ Institut für Theoretische Physik der Technischen Hochschule Braunschweig
}

(Z. Naturforschg. 19 a, 1273-1276 [1964] ; eingegangen am 26. Juni 1964)

\begin{abstract}
The penetration of a magnetic field into an infinite metallic half-space is altered due to the presence of the magnetoresistance of the medium. MAxweLL's equations modified by the magnetic field dependence of the conductivity are solved for the case where the field at the surface is switched on immediately and is constant afterwards. It is shown that due to magnetoresistance the field runs into the medium quicker compared to the case of the pure normal skin effect. The induced eddy current has its maximum value no longer at the surface of the specimen. The results are discussed in connection with pulse methods for the measurement of magnetoresistance in high fields.
\end{abstract}

Zur Messung der magnetischen Widerstandsänderung in starken Feldern verwendet man Impulsmethoden ${ }^{1}$. An der Probenoberfläche liegt ein zeitlich veränderliches Feld, das in die Probe eindringt und dort orts- und zeitabhängig ist. Die Formeln des reinen Skin-Effekts aus der Maxwellschen Theorie sind nicht anwendbar, da die spezifische Leitfähigkeit $\sigma$ eine Funktion des Magnetfeldes ist, dessen genaues Verhalten gerade durch das Vorhandensein der zu messenden magnetischen Widerstandsänderung gegenüber dem Fall des reinen Skin-Effekts modifiziert wird.

Bei der Durchführung der Experimente interessiert in diesem Zusammenhang die Wirbelstromaufheizung und die Zeit, die das Magnetfeld benötigt, um über dem Probenquerschnitt praktisch konstant zu sein. Wir wollen an einem vereinfachten mathematischen Modell zeigen, daß gerade auf Grund der Existenz einer magnetischen Widerstandsänderung das Magnetfeld schneller in die Probe eindringt. Ferner wird gezeigt, da $\beta$ das Maximum des durch das Magnetfeld induzierten Stromes immer im Innern der Probe liegt.

* Jetzt: Laboratories RCA Ltd., Zürich, Schweiz.

1 P. Kapitza, Proc. Roy. Soc., Lond. A 123, 292 [1929]. B. Lürнi, Helv. Phys. Acta 33, 161 [1960].
Zur Lösung dieses Problems ist das gekoppelte System aus Maxwellschen Gleichungen und BoLtzManN-Gleichung zu lösen:

$$
\begin{gathered}
\operatorname{rot} \boldsymbol{H}=\frac{4 \pi}{c} \boldsymbol{J} ; \quad \operatorname{rot} \boldsymbol{F}=-\frac{1}{c} \dot{H}, \\
\frac{\partial f}{\partial t}+\frac{e}{\hbar}\left\{\boldsymbol{F}+\frac{1}{c} \boldsymbol{v} \times \mathbf{H}\right\} \cdot \operatorname{grad}_{k} f \\
+\boldsymbol{v} \cdot \operatorname{grad}_{r} f=-\frac{f-f_{0}}{\tau} .
\end{gathered}
$$

Dazu kommt der Ausdruck für die Stromdichte

$$
\boldsymbol{J}=\frac{e}{4 \pi^{3}} \int \boldsymbol{v} f \mathrm{~d} \boldsymbol{k} .
$$

Da die bei den Experimenten verwendeten Frequenzen sehr klein sind gegenüber der reziproken Relaxationszeit der Elektronen $(1 / \tau)$, kann man in der Boltzmann-Gleichung (2) das Glied $\partial f / \partial t$ vernachlässigen. Ferner spielt die explizite Ortsabhängigkeit der Verteilungsfunktion $f$ nur eine untergeordnete Rolle, da sich auf der mittleren freien Weglänge $l=\tau v_{0}$ die Feldstärken nicht stark ändern. Daher ist das Glied $\boldsymbol{v} \cdot \operatorname{grad}_{\boldsymbol{r}} f$ als eine kleine Korrektur anzusehen, und man darf mit einem lokalen OHMschen Gesetz $\boldsymbol{J}=\sigma(\boldsymbol{H}) \boldsymbol{F}$ rechnen. Die Leitfähigkeit ist also als eine bekannte Funktion von $\boldsymbol{H}$ anzusehen, wie sie etwa auch bei einem ortsunabhängigen Problem vorliegt. Nicht bekannt ist jedoch die 